\title{
New faunistic records and remarks on Dermestidae (Coleoptera) - Part 20.
}

\author{
JiŘí Háva ${ }^{1,2} \&$ ANDREAS HERRMANN ${ }^{3}$ \\ ${ }^{1}$ Daugavpils University, Institute of Life Sciences and Technology, \\ Department of Biosystematics, Vienības Str. 13, Daugavpils, LV - 5401, Latvia \\ 2Private Entomological Laboratory and Collection, \\ Rýznerova 37, CZ - 25262 Únětice u Prahy, Praha-západ, Czech Republic \\ e-mail: jh.dermestidae@volny.cz \\ ${ }^{3}$ Bremervörder Strasse 123, D-21682, Stade, Germany \\ e-mail: herrmann@coleopterologie.de
}

Háva, J. \& Herrmann, A.: New faunistic records and remarks on Dermestidae (Coleoptera) - Part 20.

Abstract: The three species Globicornis cypriensis Háva \& Herrmann, 2014 stat. nov., Thorictus westwoodi Wollaston, 1854 stat. nov., Dermestes (Dermestes) cernyi Háva, 2009 stat. nov. are moved to new status. Two new synonyms are proposed: Dermestes (Dermestes) palmi Sjöberg, 1950 (= Dermestes hoeltzermanni Kozminykh, 1998 syn. nov.); Anthrenus (Anthrenus) bilyi Háva, 2000 (= Anthrenus montanus Zhantiev, 2009 syn. nov.). Fauvel. Corrections to Holloway's paper (2019) on Spanish Dermestidae are provided. 41 taxa are new records for the local fauna all over the World.

Keywords: taxonomy, new state, new synonymy, faunistics, new records, Coleoptera, Dermestidae

\section{Introduction}

The knowledge of the distribution of many species within the family Dermestidae is still incomplete. There are a lot of gaps in our understanding of the occurrence of many species. Thus, each paper, which supplements our knowledge in this field, is valuable. In the present paper, 41 species belonging to the family Dermestidae (Coleoptera) are recorded and discussed. The paper is a continuation of a series of previous papers (e. $\mathrm{g}$. Háva \& Herrmann 2016, 2018, 2019a,b).

\section{Material and methods}

Species are arranged in alphabetical order, the nomenclature and zoogeography follow the catalogue of Háva (2015). 
The following abbreviations refer to the collections, in which the examined materials are deposited:

AHEC: Andreas Herrmann, private collection, Stade, Germany;

BMNH: Natural History Museum, London, United Kingdom [British Museum (Natural History)];

ISNB: Institut Royal des Sciences Naturelles de Belgique, Brussels, Belgium;

JHAC: Jiří Háva, Private Entomological Laboratory \& Collection, Únětice u Prahy, Prague-west, Czech Republic;

MZHF: Finnish Museum of Natural History, Helsinki, Finland;

NMPC: National Museum, Praha, Czech Republic;

QCAZ: Museum of the Pontifical Catholic University of Ecuador, Ecuador;

RGPC: Rolando Salinas Gómez, private collection, Santiago, Chile;

SMNS: Staatliches Museum für Naturkunde, Stuttgart, Germany;

URPC: Uno Roosileht, private collection, Tallin, Estonia;

WZPC: Wolfgang Ziegler, private collection, Rondeshagen, Germany.

\section{Corrections to the checklist of the Spanish Dermestidae}

Holloway et al. (2019) have published a paper containing a checklist of the Spanish Dermestidae. In the paper, two species are mentioned as being valid taxa, but both are synonyms. Furthermore several relevant citations concerning synonymy and records are missing in that paper.

Holloway et al. (2019)

Paranovelsis incognitus (Háva, 2003)

Syn. Háva \& Herrmann 2018.

Anthrenus amandae Holloway, 2019

Syn. Háva \& Herrmann 2019 b. valid species

Paranovelsis aequalis (Sharp, 1902)

Anthrenus pimpinellae (Fabricius, 1775)

\section{Taxonomy}

Dermestes (Dermestes) cernyi Háva, 2009 stat. nov.

Dermestes (Dermestes) bicolor cernyi Háva, 2009: 120.

Material examined: S. Kazakhstan, Chulakkurdan env., 480 m, 25.5.2016, light, leg. Martin Marík, 1 + , A. Herrmann det., (AHEC); Kazakhstan, Almaty region, Uygur district, Temirlik canyon 43.358681, 79.165506, 2.7.2019, alt. 970m, Juha Salokannel leg., 1 ㅇ, A. Herrmann det., (AHEC); Kazakhstan, Janybek, 15.04.1996, V. Perepel leg., 1 ô, J. Háva det., (JHAC); Kazakhstan, Prov. Almaty, 22 km N Masak, 450 m, 78²7’E $43^{\circ} 13^{\prime} \mathrm{N}, 21 . v i .1996,1$ ㅇ, J. Háva det., (JHAC).

Remarks. The species was described by Háva (2009) from Uzbekistan as subspecies of D. bicolor Fabricius, 1781. Based on the characters figured by (Háva 2009) and of the recently collected specimens, the taxon is moved into a new status.

Distribution. Species known from Uzbekistan, new record for Kazakhstan. 


\section{Dermestes (Dermestes) palmi Sjöberg, 1950}

Dermestes geltzermani Kozminykh, 1997: 77.

Dermestes hoeltzermanni Kozminykh, 1998: 176 syn. nov.

Remarks. KozminykH (1997) described the species Dermestes geltzermani (holotype specimen). The same specimen has been described by KoZMINYKH (1998) as Dermestes hoeltzermanni. The species name hoeltzermanni is a new objective synonym of $D$. geltzermani respectively for D. palmi Sjöberg, 1950.

Distribution. Species known from Finland, Sweden, Mongolia, Russia: Altay, Amur, Baykal, Karelia, Khabarovsk, Perm, Primorski, Udmurtia, Ussuri.

\section{Anthrenus (Anthrenus) bilyi Háva, 2000}

Anthrenus montanus Zhantiev, 2009: 1404 syn. nov.

Material examined: Holotype of Anthrenus montanus (ठ): Armenia, Talinskij r-n, selo Karmraszen, 2005 m, 25.vi.1976, V. Gorbatovskij, (ZMAS).

Holotype of Anthrenus bilyi Háva (đ̋): Kirgizia, Kuk-kul ozero, Chamza-Abad env., 7.v.1977, Sv. Bílý lgt., (JHAC).

Remarks. Checking of type material, the species A. montanus is in all morphological characters (forming scales on pronotum and elytra, antennae, male genitalia) identical with Anthrenus (Anthrenus) bilyi Háva, 2000; A. montanus is new junior synonym of $A$. bilyi Háva.

Distribution. Species known from Armenia, Kyrgyzstan and Uzbekistan.

Globicornis cypriensis Háva \& Herrmann, 2014 stat. nov.

Globicornis signatipennis cypriensis Háva \& Herrmann, 2014: 176.

Remarks. The species was described by Háva \& Herrmann (2014) from Cyprus as subspecies of G. signatipennis Pic, 1899. Based on the characters figured by (Háva \& Herrmann 2014) and of the recently collected specimens, the taxon is moved into a new status.

Distribution. Species known from Cyprus.

Thorictus westwoodi Wollaston, 1854 stat. nov. (Fig. 1)

Thorictus westwoodi Wollaston, 1854: 220.

Thorictus grandicollis ssp. westwoodi: John, 1963: 204; Háva, 2015: 50.

Material examined: P - Porto Santo, Pico Ana Ferreira, 203 m, 330. $02.45^{\prime} \mathrm{N}$, $16^{\circ} 22.11^{\prime} \mathrm{W}, 14.10 .2018$, J. Větrovec lgt., 1 spec., J. Háva det., (JHAC).

Remarks. The species was described by Wollaston (1854) from Madeira. JoHN (1963) stated it as a subspecies of T. grandicollis Germar, 1842. Based on the characters of recently collected specimens, the taxon is moved to a new status.

Distribution. Species known from Madeira and Porto Santo Islands. 

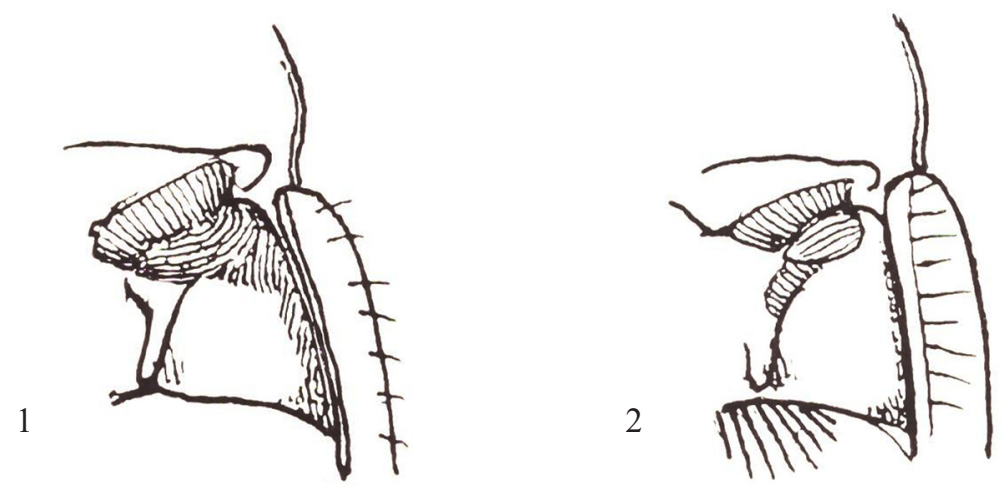

Figs. 1-2: Setation on margin of thorax, ventral view: 1- T. westwoodi Wollaston, 1854; 2- T. grandicollis Germar, 1842 (by JoHN 1963)

Faunistics

\section{Subfamily Attageninae}

Attagenus beali Zhantiev, 2005

Material examined: Yemen, Hadbramaut, As Sawm-Qbar Hud, 22.iv.1992, R. Linnavuori leg., 1 q, J. Háva det., (MZHF).

Distribution. Species known from Saudi Arabia, the United Arab Emirates, Oman, new for Yemen.

\section{Attagenus brunnescens (Pic, 1904)}

Material examined: Cyprus occ., Panagia vill., $50 \mathrm{~km}$ N of Pafos, 1100 m, 1.6.1999, F. Kantner lgt., 1 §, 1 q, J. Háva det., (JHAC).

Distribution. Species known from Armenia, Iran, Syria and Turkey, new for Cyprus.

\section{Attagenus brunneus Faldermann, 1835}

Material examined: N India, Kashmir, Srinagar Distr., Srinagar botanical garden,

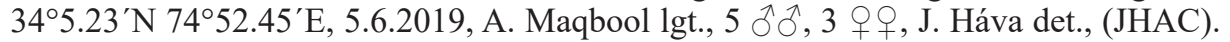

Distribution. Holarctic species, new for India: Kashmir.

Attagenus cyphonoides Reitter, 1881

Material examined: Ukraine, Zhytomyr Raion, Zhytomyr city [130 km W of Kyev], 6.2019, O. Masliukivskyi lgt., 1 §̃, J. Háva det., (JHAC).

Distribution. Species known from Europe, Egypt, Morocco, Nigeria, Senegal, South Sudan, Sudan, Tunisia, Afghanistan, China, India, Iran, Iraq, Israel, Kazakhstan, Mongolia, Pakistan, Russia, Saudi Arabia, Tajikistan, Turkmenistan, Uzbekistan, Canada, Mexico, U.S.A., Australia: Queensland, new for Ukraine. 


\section{Attagenus smirnovi Zhantiev, 1973}

Material examined: Russia, Ussuri Reg., 20 km NW Chernyshevka, Anuchinsky distr., 200 m, 11-18.8.2016, A. Shamaev lgt., 1 đ̃, J. Háva det., (JHAC).

Distribution. Species known from Palaearctic, Oriental and Afrotropical Regions, new for Russia: Ussuri Region.

\section{Attagenus unicolor unicolor (Brahm, 1790)}

Material examined: Uruguay, Montevideo, Penarol, 20.12.1930, 1 §ै, A. Herrmann det., (AHEC).

Distribution. Cosmopolitan species, new for Uruguay.

\section{Attagenus unicolor simulans Solsky, 1876}

Material examined: N India, Kashmir, Srinagar Distr., Srinagar botanical garden, $34^{\circ} 5.23^{\prime} \mathrm{N} 74^{\circ} 52.45^{\prime} \mathrm{E}, 5.6 .2019$, A. Maqbool lgt., 1 §̋, 12 우, J. Háva det., (JHAC).

Distribution. Species known from Afghanistan, N China, Kazakhstan, Kyrgyzstan, Mongolia, Russia, Tajikistan, Turkmenistan, Uzbekistan, new for India: Kashmir.

\section{Subfamily Dermestinae}

\section{Dermestes (Dermestinus) szekessyi szekessyi Kalík, 1950}

Material examined: Estonia, Tehumardi, $58^{\circ} 10^{\prime} / 22^{\circ} 15^{\prime}, 15.07 .2018$, seashore under seaweed, U. Roosileht lgt., 1 spec., U. Roosileht \& A. Herrmann det., (URPC).

Distribution. Species known from Europe, C, W Kazakhstan, Mongolia, Russia: Astrakhan, Novosibirsk, Stavropol, Tuva (Háva 2015), new for Estonia.

\section{Dermestes (Dermestinus) undulatus Brahm, 1790}

Material examined: Belge Congo, Bambesa, 10.vi.1937, J. Vrydagh, 1 spec., A. Herrmann det., (ISNB).

Distribution. Holarctic species, new introduced species for Congo.

\section{Dermestes (Montandonia) fuliginosus Rossi, 1792}

Material examined: Armenia, Aragatsotn, Pamb Pass, 2152 m, 5.6.2017, W. Ziegler leg., 1 $\hat{\jmath}$, A. Herrmann det., (WZPC).

Distribution. Species known from Europe, Azerbaijan, Georgia, Turkey, Iran, W Russia, new for Armenia. In the literature (HÁva 2015), this species is recorded also from „Caucasus“.

\section{Subfamily Megatominae}

Anthrenus (Anthrenodes) amoenulus Reitter, 1896

Material examined: Cyprus, Paphos, Yeroskipos, 20.7.1939, Hakar Linb., 3 spec., J. Háva det., (2 MZHF, 1 JHAC); Cyprus, Kambos, 25.7.1939, Hakar Lindb., 1 spec., J. Háva det., (MZHF); Cyprus, Larnaka, 25.6.-1.7.1939, Hakar Lindb., 1 spec., J. Háva det., (MZHF); Cyprus, Kykko, 15-17.7.1939, Hakar Lindb., 2 spec., J. Háva det., (MZHF, JHAC). 
Distribution. Species known from Afghanistan, Caucasus, Caspian Lowlands, Iran, Russia, Turkey, Turkmenistan, new for Cyprus.

Anthrenus (Anthrenodes) fernandezi Háva, 2003

Material examined: Chad, Farcha, 20-22.5.1973, R. Linnavuori leg., 1 q, J. Háva det., (MZHF); Nigeria Kw. St., Shangunu-Rofia, 22.7.1973, R. Linnavuiro leg., 2 우, J. Háva det., (MZHF, JHAC).

Distribution. Species known from Burkina Faso, Cameroon, new for Chad and Nigeria.

Anthrenus (Anthrenus) pimpinellae isabellinus Küster, 1848

Material examined: Israel c., Old Yaffa port, $32^{\circ} 05^{\prime} \mathrm{N} 34^{\circ} 75^{\prime} \mathrm{E}, 24 . i v .2019$, V. Novák lgt., 1 spec., J. Háva det., (JHAC).

Distribution. Species known from France, Italy, Spain, Algeria, Morocco, Tunisia, U.S.A. (introduced), new for Israel.

\section{Anthrenus (Anthrenus) pimpinellae pimpinellae (Fabricius, 1775)}

Material examined: England, London, Battersea Park, 20.6.2015, L. Roučka leg., 1 ô, 2 우, J. Háva et., (JHAC).

Remarks. Holloway et al. (2018) removed this species from the British fauna. DufF (2018) mentioned this species in the list of British Coleoptera. Herewith the occurrence is confirmed for England.

Distribution. Nearly cosmopolitan species.

Anthrenus (Anthrenus) scrophulariae (Linnaeus, 1758)

Material examined: Argentina, Tucuman, Tafi del Valle, ii. 1956, Col. C. Orlog, 1 spec., A. Herrmann det., (AHEC).

Distribution. Nearly cosmopolitan species, new introduced species to Argentina.

\section{Anthrenus (Anthrenus) senegalensis Pic, 1927}

Material examined: Benin (West-Afrika): Natilingou, 13.4.1988, K. Erger leg., 2 $ぇ$, J. Háva det., (SMNS, JHAC).

Distribution. Species known from Gambia, Guinea Bissau, Senegal, Sierra Leone, new for Benin.

Anthrenus (Nathrenus) biskrensis Reitter, 1887

Material examined: I. Lipari, Vallone Muria, 19.vi.1998, B. Carleti, B. Cecchi, L. Dapporto, P Lo Cascio, C. Moreno leg., 1 spec., J. Háva det., (JHAC).

Distribution. Species known from Italy: Lampedusa, Sicily, Malta, Spain, Algeria, Libya, Morocco, Tunisia, new for Italy: Lipari Island. 


\section{Anthrenus (Nathrenus) zahradniki Háva, 2003}

Material examined: „Cypr. [Cyprus], Kykko, 15-17.7.1939, Hakan Lindb.”, 2 spec., J. Háva det., (MZHF).

Distribution. Species known from Greece, Lebanon, Turkey, new for Cyprus.

Cryptorhopalum bicolor Sharp, 1902

Material examined: Ecuador, Napo, Cosanga, 2100 m, malaise trap, 9.2019, Jim McClarin leg., 1 spec., J. Háva det., (QCAZ).

Distribution. Species known from Belize, Honduras, Nicaragua, Panama, new for Ecuador.

\section{Cryptorhopalum germanum Sharp, 1902}

Material examined: Mexico: Mexico state, $40 \mathrm{~km}$ NW Puebla de Zaragoza, $2800 \mathrm{~m}$, $19^{\circ} 35^{\prime}$ N, 9866’W, 26.vi.2019, A. Shamaev leg., 8 spec., J. Háva det., (JHAC).

Distribution. Species known from Guatemala, Mexico: Guanajuato, Guerrero, Morelos, Oaxaca, new for Mexico: Mexico state.

Orphinus (Orphinus) beali Herrmann, Háva \& Zhang, 2011

Material examined: China, Shaanxi, Qinling mts., Xunyangba (6 km E), 1000-1300 m, 23.v.-13.vi.1998, I. H. Marshal lgt., 1 †, J. Háva det., (JHAC); China, Sichuan prov., Ya'an prefecture, Ya'an Co., Road 108, ca. 20 km S Ya'an, ca. 1000 m, 19.vi.1998, A. Pütz leg., 1 §̃, J. Háva det., (JHAC).

Distribution. Species known from China: Yunnan, new for Shaanxi and Sichuan.

\section{Orphinus (Orphinus) bezdeki Háva \& Kadej, 2016}

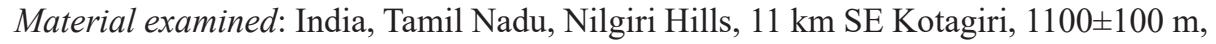
Kunchapanai env., $11^{\circ} 22^{\prime} \mathrm{N} 76^{\circ} 56^{\prime} \mathrm{E}, 7-22 . v .2000$, P. Pacholátko leg., 51 spec., (47 NMPC, 4 JHAC).

Distribution. Species known from India: Maharasthra, new for India: Tamil Nadu.

Orphinus (Orphinus) kadeji Herrmann \& Háva, 2014

Material examined: Laos, Vientiane / 30.iii.1990, E. Kondorosy leg., 1 †, J. Háva det., (JHAC).

Distribution. Species known from Thailand, new for Laos.

Orphinus (Orphinus) kresli Háva, 2003

Material examined: Nepal W, Gorkha, Khanchok-Arughat Bazar, 1300-1700 m, 23.5.1990, Probst leg., 1 o, J. Háva det., (JHAC).

Distribution. Species known from central Nepal, otherwise second known specimen. 
Orphinus (Orphinus) sikkimensis Háva \& Herrmann, 2004

Material examined: Bhutan west, Thimphu Prov., Taba, 2500-2600 m, 15-22.viii.1990, C. Holzschuh leg., 1 q, J. Háva det., (JHAC).

Distribution. Species known from India: Sikkim, Darjeeling, new for Bhutan.

Orphinus (Orphinus) unifasciatus Háva, 2006

Material examined: „India, Bhimthal [Uttaranchal], ii. [19]86”, 1 q, J. Háva det., (JHAC).

Distribution. Species known from India: Himachal Pradesh, new for Uttaranchal. Second known specimen.

Phradonoma charon Háva \& Kadej, 2008

Material examined: Cyprus, Limassol, Colossi, 3.7.1939, Hakkan Lindb., 1 spec., J. Háva det., (MZHF).

Distribution. Species known from Greece, Turkey, new for Cyprus.

Reesa vespulae (Milliron, 1939)

Material examined: „RA.Neuquen, 26.ii.1946, Hayward. Willink“, 1 spec., A. Herrmann det., (AHEC).

Distribution. Cosmopolitan species, new for Argenitina.

Thaumaglossa dembickyi Háva, 2002

Material examined: Philippines, Central Visayas, Samar, Lope de Vega, Febr. 2019, local collector, 1 §, 1 ㅇ, J. Háva det., (JHAC); Philippines, MINDANAO, Zamboanga del Norte, Gutallac, March 2019, local collector, 1 ô, J. Háva det., (JHAC).

Distribution. Species already known from the Philippines (HÁvA 2015): Luzon, Mindanao, new for Visayas.

Thaumaglossa rufula Pic, 1931

Material examined: Indonesia, E Kalimantan, Maura Ritan vill., $00^{\circ} 24.0^{\prime} \mathrm{N}, 116^{\circ} 1^{\prime} \mathrm{E}$, 49 m, 5. Xii.2011, J. Hájek, J. Schneider \& P. Votruba leg. / border of fields and rainforest in foothill near Beleyan river, individual collecting in puddle and on vegetation, + light trap, 1 đ, J. Háva det., (NMPC).

Distribution. Species known from the Philippines, Indonesia: Java, new for Kalimantan.

Trogoderma diiorioi Háva, 2017

Material examined: Argentina, Bariloche/Rio Negro, 01.01.1955, Leg. S. Schajovskoi, 1 spec., A. Herrmann \& J. Háva det., (AHEC); Argentina, Pucará/Parque Nacional Lanín, XI-1951, Leg. S. Schajovskoi, 1 ふ̃, A. Herrmann \& J. Háva det., (AHEC). Distribution. Species known only from Argentina. 
Trogoderma koenigi Pic, 1954

Material examined: China, Zhejiang prov., West Tianmu Shan (Mts.) reserve, border of secondary mixed forest near entrance of reserve; at light, $30^{\circ} 18.34^{\prime} \mathrm{N}, 119^{\circ} 26.46^{\prime} \mathrm{E}$, 310 m, 23.vi.-6.vii.2017, J. Hájek \& J. Růžička leg., 1 ㅇ, J. Háva det., (NMPC).

Distribution. Species known from China: Fujian, new for Zhejiang Prov.

Trogoderma stachi Mroczkowski, 1958

Material examined: „Buenos Aires, I.30“, 1 §ૈ, J. Háva det., (AHEC); „Sao Paulo, Bras.“/ „Eurhopalus ? vicinus Sol var.“ [M. Pic. det], 1 §, J. Háva det., (JHAC).

Remarks. The habitus and male genitalia are firstly illustrated (Figs. 3-4).

Distribution. Species known from Brazil, new for Argentina.
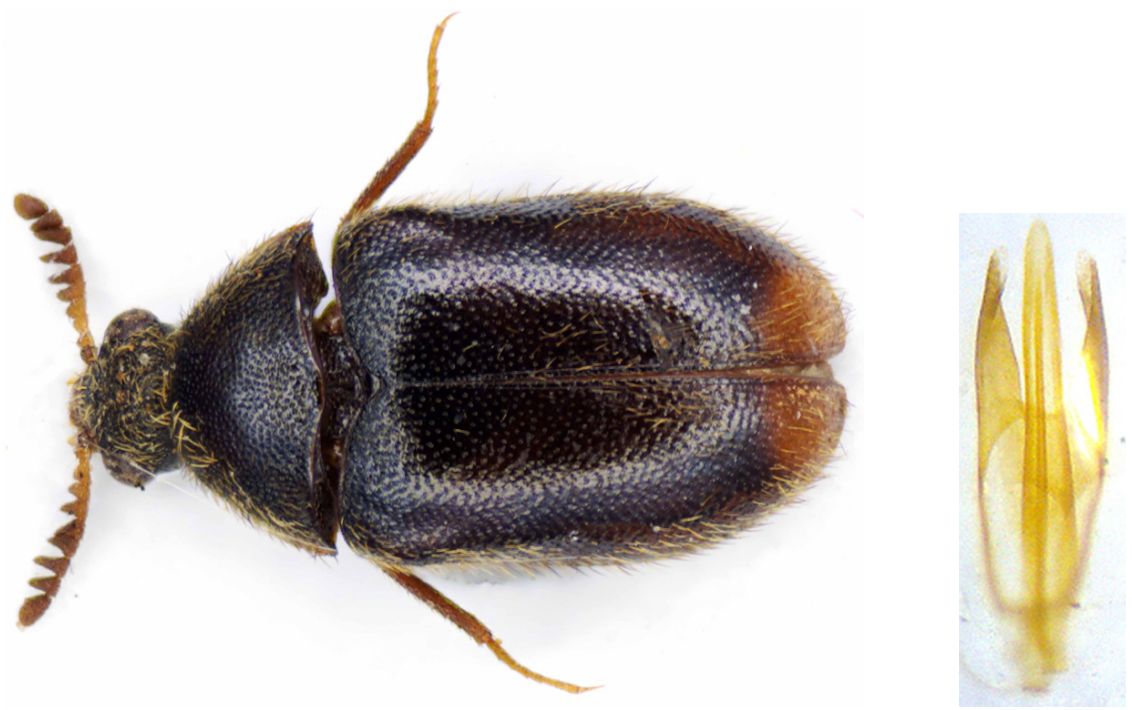

Figs. 3-4: Trogoderma stachi Mroczkowski, 1958: 3- habitus, dorsal view; 4- male genitalia

Trogoderma variabile Ballion, 1878

Material examined: Argentina, Mendoza, Luján de Cuyo en plafón, 15.01.2019, leg. G. Gomariz, 1 + , A. Herrmann det., (AHEC).

Distribution. Nearly cosmopolitan species, newly introduced species to Argentina.

\section{Subfamily Orphilinae}

\section{Orphilus subnitidus LeConte, 1861}

Material examined: Mexico, Baja California state, Parque Nac. Constitution de 1857, 12 km SW Laguna Hanson, 1700 m, Sierra de Juares, 31.96N 1.5.99W, 15.vi.2019, A. Shamaev leg., 5 spec., J. Háva det., (JHAC).

Distribution. Species known from Canada: Alberta, British Columbia, West U.S.A, new for Mexico: Baja California. 


\section{Subfamily Thorictinae}

Thorictus grandicollis grandicollis Germar, 1842

Material examined: Chile, Región de O'higgins (VI Región), Cerro Llivi llivi, Lo Miranda, 13.10.2019, leg. Rolando Salinas Gómez, 1 spec., J. Háva \& A. Herrmann det., (RGPC).

Remarks. The entomologist Rolando Salinas Gómez from Chile informed us by email that he found a specimen of the genus Thorictus near Santiago, in the coastal mountain range. It walked through some wooden sticks in one of his terrariums; the specimen probably arrived with some Camponotus chilensis that he caught a few days before. This was the first record of a specimen belonging to the genus Thorictus from America as well as from the whole Neotropical region. The species is unlikely to have been hiding in America to this day, the beetle was certainly introduced from Europe, Africa or Asia. The genus Thorictus includes more than 170 valid species respectively subspecies, all of them are very small, entirely brown or black, nearly hairless and look habitually very similar to each other. Thorictus grandicollis Germar, 1842 is the most common species of this genus in Southern Europe and the Mediterranean subregion.

Distribution. The species known from south Europe and the Medditerranean subregion, Armenia, Azerbaijan and Turkmenistan, newly introduced in Chile.

\section{Acknowledgements}

We are indebted to all colleagues and collectors for the chance to study their material. 


\section{References}

DufF, A. G. 2018: Checklist of Beetles of the British Isles. - 3rd Edition. Iver: Pemperley Books, 248 pp.

Háva, J. 2009: Dermestidae (Coleoptera) from the collection of the Czech entomologist Zdeněk Černý. - Baltic Journal of Coleopterology 9: 119-123.

HÁva, J. 2015: World Catalogue of Insects. Volume 13. Dermestidae (Coleoptera). - Leiden/Boston: Brill, xxvi $+419 \mathrm{pp}$.

Háva, J. \& Herrmann, A. 2014: A new subspecies of Globicornis (Coleoptera: Dermestidae: Megatominae) from Cyprus. - Arquivos Entomolóxicos 12: 175-177.

Háva, J. \& Herrmann, A. 2016: New faunistic records and remarks on Dermestidae (Coleoptera) - Part 16. - Folia Heyrovskyana, Series A 25(2): 4-14.

Háva, J. \& Herrmann, A. 2018: New faunistic records and remarks on Dermestidae (Coleoptera) - Part 17. Folia Heyrovskyana, Series A 26(1): 13-18.

Háva, J. \& Herrmann, A. 2019a: New faunistic records and remarks on Dermestidae (Coleoptera) - Part 18. - Studies and Reports, Taxonomical Series 15(1): 41-53.

Háva, J. \& Herrmann, A. 2019b: New faunistic records and remarks on Dermestidae (Coleoptera) - Part 19. - Natura Somogyiensis 33: 27-36.

Holloway, G. J., Foster, Ch. W. \& Barclay, M. V. L. 2018: Anthrenus piminellae Fabricius, 1775 (Coleoptera: Dermestidae): the case for removal from the British list. The Coleopterist 27(2): 74-76.

Holloway, G. J., Cañada Luna, I. \& Kadej, M. 2019: A review of the literature and a Checklist of the Spanish Dermestidae (Coleoptera). - The Coleopterists Bulletin 73(3): 513-520.

JoHN, H. 1963: Revision der Gattung Thorictus Germar (Thorictidae, Clavicornia Col.). Eine Bestandsaufnahme, Kritik und Neubeschreibungen. - Entomologische Abhandlungen und Berichte aus dem Staatlichen Museum für Tierkunde in Dresden 28: 177-255.

KozminyKh, V. O. 1997: Sostav fauny zhestkokrylych (Insecta: Coleoptera) semejstev Sphaeritidae, Histeridae i Dermestidae Permskoj oblasti. pp. 71-79. - In: Esjunina S. L., Zinovjeva E. A., Litvinenko N. I., Ovesnova S. A. (eds.): Problemy regionalnoj Krasnoj knigi. Mezhvedomstvennyj sbornik naucznych trudov, Permskij gosuniversitet, 152 pp. (in Russian).

KozminYKh, V. O. 1998: Spisok vidov zhestkokrylych (Insecta, Coleoptera) iz fondov Permskogo Oblastnogo Kraevedczeskogo Muzeja. In: 80 let farmacevticzeskomu obrazovaniju i nauke na Urale. - Itogi i perspektivy: materialy mezhuvovskoj nauczno-prakticzeskoj konferencii, nosbjascenoj 275-letiju goroda Permi u 80-letiju farmacevticzeskogo obrazovanija na Urale. Perm: PGFA, 241 pp. (in Russian)

Wollaston, T. V. 1854: Insecta Maderensia; being an account of the insects of the islands of the Maderian group. - London: J. van Voorst, xlii + 634 pp., 13 pls.

Zhantiev, R. D. 2009: New and little-known dermestid-beetles (Coleoptera, Dermestidae) from the Caucasus. - Zoologicheskiy Zhurnal 88(11): 1402-1405. (in Russian, English summary) 
